\title{
A Novel Dimension Reduction Technique for Physical Activity Recognition from a Waist-Mounted Accelerometer
}

\author{
Benjawan Prapochanang ${ }^{\mathrm{a}, *}$, Thurdsak Leauhatong ${ }^{\mathrm{a}}$, Nitipat Nattaitanakul ${ }^{\mathrm{a}}$ \\ ${ }^{a}$ Faculty of Engineering, King Mongkut's Institute of Technology Ladkrabang, Bangkok and 10520, Thailand \\ *Corresponding Author: benjawan_0407@hotmail.com and s5612705@kmitl.ac.th
}

\begin{abstract}
This paper proposes a novel dimension reduction technique for physical activity recognition from a waistmounted accelerometer. Firstly, the wavelet transform is used to extract features from the acceleration signals. Then the proposed technique is used to reduce the dimension of the wavelet features. Finally, the Multi-Layer Perceptron Neural Network (MLPNN) is used to recognize the physical activities from the reduced features. In our experiments, 5 volunteers who were healthy with the ages between 21 to 25 year old were asked to mount a tri-axial accelerometer at the right side of their waists. Next, the volunteers were asked to perform 5 daily-life physical activities: 1) walking 2) standing up from a chair 3) sitting down on a chair 4) lying down on a bed and 5) getting up from a bed; and 5 falling events: 1) forward fall 2) backward fall 3) falling to the right side 4) falling to the left side and 5) falling when standing up. In order to evaluate the performance of the proposed algorithm, the precision of the recognition and the total number of the used operators were used. The performance of the recognition with different setting of mother wavelets, vanishing moments, the rate of the dimension reduction, and the number of nodes of the hidden layer were evaluated. From the experiments, the proposed dimension reduction not only reduce the total number of the used operators but also increase the precision of the recognition.
\end{abstract}

Keywords: physical activity recognition, wavelet transform, dimension reduction, neural network.

\section{Introduction}

From the report of World Population Ageing ${ }^{(1)}$, the number of elderly people have been increasing. Falls are their most dangerous problem which causes hip fractures, disabilities, or accidental deaths. For example in USA from 1999 to 2010, the elderly mortalities due to fall-related injuries were reached up to $186,029^{(2)}$. The falls may be resulted from 2 major causes: 1) physical problems, such as knee surgery, degenerative arthritis, hypertension, or heart disease; and 2) environment problems, such as floor slippery or rough, and level or slope path. In order to reduce the rate of the mortalities or injuries, a system which can predict or detect the falls efficiently and accurately has to be developed.

From the advances of miniature technologies, inertia wearable sensors such as accelerometers and gyroscopes are widely used to monitor human movements in daily life. Their advantages are cost efficiency, light weight, small size, low power consumption, and convenience in the independent living environment ${ }^{(3)}$. Many researchers have developed algorithms to distinguish daily-life activities from falling events. For example, Y. Li, et al. ${ }^{(4)}$ proposed the fall detection method based on Neyman-Pearson detection framework. From the experimental results, the performances obtained by the training data and testing data show a good matching.

In this paper, we proposed an algorithm for physical activity recognition from a waist-mounted accelerometer. The algorithm consists of 3 steps; 1) wavelet transform, 2) the proposed dimension reduction, and 3) physical activity recognition.

\section{The Signal Acquisition Device}

Our signal acquisition device consists of a tri-axial accelerometer (MMA7331L) and a microcontroller with a 10-bit analog-to-digital convertor. The device is mounted to the right side of the waist of the volunteer subjects, and the orientation of the three axes is arranged as shown in Fig. 1.

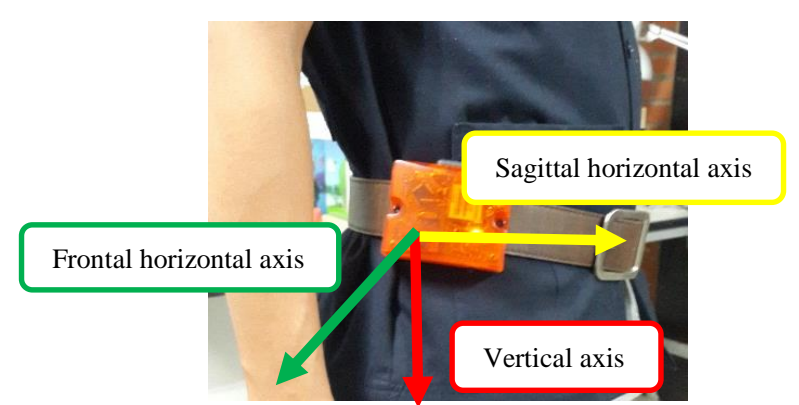

Fig. 1. The orientation of the three axes of the accelerometer.

From our observations, the frequency range of the acceleration signals of all physical activities and falling events were from 4 to $100 \mathrm{~Hz}$. As a result, the sampling rate of three acceleration signals are set to $200 \mathrm{~Hz}$. According to the experiments of Sengto and Leauhatong ${ }^{(5)}$, a full-scaled 
affection of the accelerometer is set to $+/-4 G$ (where $G$ represents acceleration due to gravity: $9.81 \mathrm{~m} / \mathrm{s}^{2}$ ).

From our observations, all of the falling signals can be separated into three parts. The first part is the acceleration signals of some activities that cause the fall such as slip, stumble, sway, and etc. Usually, this part takes one second. The second part is the acceleration signals that is occurred when some parts of body, such as hand, hip, knee, or head, impact the ground. Since the impacts usually cause rapid change of the acceleration of the body, most of the impact signals have one or two peaks within 100-200 millisecond. The third part is the signals of some activities that occur after the impact such as roll or bounce of the body. This part usually takes less than one second. Then we assume that most of the falling activities take 2 seconds.

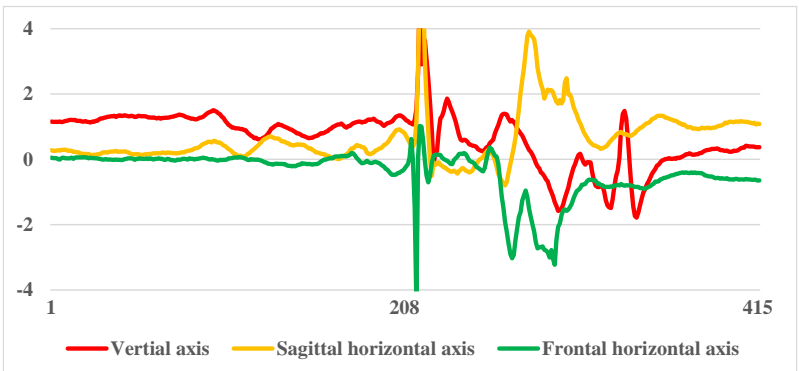

Fig. 2. An example of the acceleration signals of vertical -, sagittal horizontal -, and frontal horizontal axes of a forward falling.

According to the aforementioned, the lengths of three acceleration signals are set to 416 samples. Then the total number of the samples used in the proposed algorithm is 1248 samples. An example of the acceleration signal of vertical -, sagittal horizontal -, and frontal horizontal - axes of a forward falling are shown in Fig.2.

\section{The Physical Activities Recognition}

The recognition can be separated into three processes; 1) feature extraction, 2) dimension reduction, and 3) activity recognition. The details of three processes are described as follows.

\subsection{The Wavelet Feature Extraction}

In the wavelet transform theory ${ }^{(6,7)}$, a signal can be constructed from its approximation and details as follows:

$$
x(t)=\sum_{k=0}^{2^{N-j}-1} a_{j, k} 2^{-\frac{j}{2}} \phi\left(2^{-j} t-k\right)+\sum_{j=1}^{j} \sum_{k=0}^{2^{N-j}-1} d_{j, k} 2^{-\frac{j}{2}} \psi\left(2^{-j} t-k\right),
$$

where $\phi$ and $\psi$ are scaling and wavelet functions respectively. $a$ and $d$ are approximate and detailed coefficients of $\phi$ and $\psi$ functions respectively. $j$ and $k$ are integer values which represent dilation and translation parameters of the transform. $a$ and $d$ coefficients can be calculated as follows:

$$
\begin{aligned}
& a_{j, k}=\left\langle x(t), \phi_{j, k}(t)\right\rangle=\sum_{n} h(n-2 k) a_{j-1, n}, \\
& d_{j, k}=\left\langle x(t), \psi_{j, k}(t)\right\rangle=\sum_{n} g(n-2 k) a_{j-1, n},
\end{aligned}
$$

where $h(n)$ is the low-pass filter of scaling function, and $g(n)$ is the high-pass filter of wavelet function. These filters are constructed from the selected wavelet function and its corresponding scaling function as follows:

$$
\begin{aligned}
& \phi_{j, k}=\sqrt{2} \sum_{n} h(n) \phi(2 t-n), \\
& \psi_{j, k}(t)=\sqrt{2} \sum_{n} g(n) \phi(2 t-n) .
\end{aligned}
$$

Table 1. Properties of different mother wavelets.

\begin{tabular}{|c|c|}
\hline WAVELET FAMILY & FILTER LENGTH \\
\hline Daubechies (db-N) & $2 \mathrm{~N}$ \\
Coiflets (coif-N) & $6 \mathrm{~N}$ \\
Symlets (sym-N) & $2 \mathrm{~N}$ \\
Biorthogonal (bior-Nr/Nd) & $\max (2 \mathrm{Nr}, 2 \mathrm{Nd})+2$ \\
\hline
\end{tabular}

The wavelet coefficients can be used as efficient features; however in order to extract the efficient wavelet features, the mother wavelets and their number of vanishing moments ${ }^{(8,9)}$ must be carefully selected. The number of the vanishing moments relates to the order of function that can be approximated by corresponding wavelet. For the analysis of highly transient signals with high complexity, wavelets with more vanishing moments are more suitable as they result in a more compact representation of the signals. The vanishing moments linearly relate to the filter length. Table 1 . shows the relation between the filter length and the vanishing moments of the mother wavelets used in this paper, where $N$ is the number of vanishing moments.

\subsection{The Proposed Dimension Reduction}

In our experiments, the number of the wavelet features are very large (1248 coefficients), but the number of the training acceleration signals are very small (750 signals). As a result, they lead to two well-known problems called the curse of dimensionality (COD) and the small sampling size (SSS) problem ${ }^{(10,11)}$. In order to improve both of the accuracy and the time complexity, a dimension reduction method must be used. However, most of dimension reduction techniques such as principal component analysis and linear discriminant analysis cannot be used in the SSS problem. Inspired by the $\mathrm{LDA}^{(12)}$, this paper proposed a novel technique that can solve both problems. The proposed technique can be described as follows.

Let $a_{v}, a_{s}$, and $a_{f}$ be column matrices of the approximate coefficients of the 1-level decomposition of 
the vertical-, sagittal horizontal-, and frontal horizontalaxes of an acceleration signal respectively. Let $d_{v}, d_{s}$, and $d_{f}$ be column matrices of the detailed coefficients of the 1-level decomposition of the vertical-, sagittal horizontal-, and frontal horizontal-axes of an acceleration signal respectively. Since the number of samples of each axis of the acceleration signals is 416 , then the number of coefficients of $a_{v}, a_{s}, a_{f}, d_{v}, d_{s}$, and $d_{f}$ are 208.

Let $r$ be the down sampling ratio. Approximation and detailed column matrices of the $r$ down sampling at the $i^{\text {th }}$ position can be described as follows:

$$
\begin{aligned}
& \mathbf{a}_{A x, r, i}=\left[a_{A x}(i), a_{A x}(r+i), a_{A x}(2 r+i), \ldots\right]^{T}, \\
& \mathbf{d}_{A x, r, i}=\left[d_{A x}(i), d_{A x}(r+i), d_{A x}(2 r+i), \ldots\right]^{T}
\end{aligned}
$$

where $A x \in\{v, s, f\}$, and $i \in\{0,1, \ldots, r-1\}$. As a result for a given $r$, the number of the down sampling approximation and detailed matrices is $2 \times 3 \times r$, and their dimensions are $208 / r$.

Given $A x, r$, and $i$, let $A_{A x, r, i, c}$ and $D_{A x, r, i, c}$ be respectively the sets of the down sampling approximation and detailed matrices of the $c^{\text {th }}$ activity. The mean matrices of $A_{A x, r, i, c}$ and $D_{A x, r, i, c}$ can be calculated as follows:

$$
\begin{aligned}
& \overline{\mathbf{a}}_{A x, r, i, c}=\frac{1}{n_{c}} \sum_{\mathbf{a}_{A x, r, i} \in A_{A x, r, i, c}} \mathbf{a}_{A x, r, i}, \\
& \overline{\mathbf{d}}_{A x, r, i, c}=\frac{1}{n_{c}} \sum_{\mathbf{d}_{A x, r i} \in D_{A x, r, i, c}} \mathbf{d}_{A x, r, i}
\end{aligned}
$$

where $n_{c}$ is the number of the training acceleration signals of the $c^{\text {th }}$ activity.

Given $r$ and $c$, the error matrices of the down sampling approximation and detailed matrices can be formulated as follows:

$$
\mathrm{E}_{r, c}=\left[\hat{\mathbf{a}}_{v, r, 0, c}, \ldots \hat{\mathbf{a}}_{f, r, r-1, c}, \hat{\mathbf{d}}_{v, r, 0, c}, \ldots \hat{\mathbf{d}}_{f, r, r-1, c}\right]
$$

where

$$
\begin{aligned}
& \hat{\mathbf{a}}_{A x, r, i, c}=\left(\mathbf{a}_{A x, r, i}-\overline{\mathbf{a}}_{A x, r, i, c}\right), \\
& \hat{\mathbf{d}}_{A x, r, i, c}=\left(\mathbf{d}_{A x, r, i}-\overline{\mathbf{d}}_{A x, r, i, c}\right),
\end{aligned}
$$

where $\mathbf{a}_{A x, r, i} \in A_{A x, r, i, c}$, and $\mathbf{d}_{A x, r, i} \in A_{A x, r, i, c}$.

The within-class variance scatter can be calculated as follows:

$$
\mathbf{V}_{r}^{W}=\sum_{c} n_{c} \mathrm{E}_{r, c}^{T} \mathrm{E}_{r, c}
$$

Let $N$ be the total number of the training acceleration signals. The mean error matrices of the down sampling approximation and detailed matrices can be formulated as follows:

$$
\overline{\mathrm{E}}_{r, c}=\left[\tilde{\mathbf{a}}_{v, r, 0, c}, \ldots, \tilde{\mathbf{a}}_{f, r, r-1, c}, \tilde{\mathbf{d}}_{v, r, 0, c}, \ldots, \tilde{\mathbf{d}}_{f, r, r-1, c}\right],
$$

where

$$
\begin{aligned}
& \tilde{\mathbf{a}}_{A x, r, i, c}=\overline{\mathbf{a}}_{A x, r, i, c}-\frac{1}{N} \sum_{\mathbf{a}_{A x, r, i}} \mathbf{a}_{A x, r, i}, \\
& \tilde{\mathbf{d}}_{A x, r, i, c}=\overline{\mathbf{d}}_{A x, r, i, c}-\frac{1}{N} \sum_{\mathbf{d}_{A x, r, i}} \mathbf{d}_{A x, r, i},
\end{aligned}
$$

and the between-class variance scatter can be calculated as follows:

$$
\mathbf{V}_{r}^{B}=\sum_{c} n_{c} \overline{\mathrm{E}}_{r, c}^{T} \overline{\mathrm{E}}_{r, c}
$$

The proposed dimension reduction technique is to find the new projection $\mathbf{w}$ that maximization the criteria

$$
J(\mathbf{w})=\frac{\mathbf{w}^{T} \mathbf{V}_{r}^{B} \mathbf{w}}{\mathbf{w}^{T} \mathbf{V}_{r}^{W} \mathbf{w}} .
$$

As a result, $\mathbf{w}$ is the eigenvector of the problem

$$
\operatorname{det}\left(\left(\mathbf{V}_{r}^{W}\right)^{-1} \mathbf{V}_{r}^{B}-\lambda I\right)=0
$$

The output of the proposed dimension reduction technique can be calculated as follows:

$$
\mathbf{F}_{r}=\mathbf{A}_{r} \mathbf{W}
$$

where $\quad \mathbf{A}_{r}=\left[\mathbf{a}_{v, r, 0}, \ldots, \mathbf{a}_{f, r, r-1}, \mathbf{d}_{v, r, 0}, \ldots, \mathbf{d}_{f, r, r-1}\right]$ is the input matrix of the down sampling approximation and detailed coefficients of the test acceleration signals. $\mathbf{W}=\left[\mathbf{w}_{1}, \mathbf{w}_{2}, \mathbf{w}_{3}\right]$ is the matrix of the first three eigenvector. $\mathbf{F}_{r}=\left[\mathbf{f}_{r, 1}, \mathbf{f}_{r, 2}, \mathbf{f}_{r, 3}\right]$ is the output matrix of the proposed dimension reduction. Since the number of the columns of $\mathbf{A}_{r}$ and $\mathbf{F}_{r}$ are $2 \times 3 \times r$ and 3 respectively, the dimension reduction ratio equals to $2 r$.

\subsection{Activity Recognition}

The MLPNN ${ }^{(13)}$ is used to recognize the activities. The used network consists of the input, hidden, and output layers. The activation function of the neurons are the log sigmoid, $f(x)=1 /(1+\exp (-x))$. The network was trained by the back propagation algorithm.

The wavelet features whose dimension are reduced by the proposed dimension reduction algorithm are fed to the 
input layer. Then the number of the neurons in the input layer equals to the reduced dimension. The number of the neurons in the hidden layer is defined by experiments (see in Section 4). The number of the neurons of the output layer depends on the objective of the recognition.

\section{The Performance Evaluation}

The performance of the proposed algorithm depends on its 4 factors; 1) the mother wavelets, 2) the vanishing moments (the filter length), 3) the dimension reduction ratio, and 4) the number of neurons in the hidden layer. Table 2. shows all of the selected setting of the factors used in this paper.

Table 2. The selected setting of the factors.

\begin{tabular}{|c|c|}
\hline Factors & Selected Setting \\
\hline $\begin{array}{c}\text { Wavelets and } \\
\text { Vanishing } \\
\text { Moments }\end{array}$ & $\begin{array}{l}\text { 'db2', 'db4', 'db6', ..., 'db20', 'coif1', ..., } \\
\text { 'coif5', 'sym2', 'sym3', ..., 'sym9', 'bior1.1', } \\
\text { 'bior1.3', 'bior1.5', 'bior2.2', 'bior2.4', } \\
\text { 'bior2.6', 'bior2.8', 'bior3.1', 'bior3.3', } \\
\text { 'bior3.5', 'bior3.7', 'bior3.9', 'bior4.4', } \\
\text { 'bior5.5', and 'bior6.8' }\end{array}$ \\
\hline $\begin{array}{l}\text { The dimension } \\
\text { reduction ratio }\end{array}$ & 4,8 , and 16 \\
\hline $\begin{array}{c}\text { Neurons in } \\
\text { hidden layer }\end{array}$ & $1,2,3, \ldots$, and 40 \\
\hline
\end{tabular}

The ideal system must provide high accuracy and require low time complexity. However in practical, one has to trade the accuracy for the complexity. In this paper, percent of the average precision $(\% \overline{\mathrm{Pr}})^{(14)}$ and percent of the used operators $(\% \mathrm{Op})$ are used to measure the accuracy and time complexity respectively. Moreover, we proposed a new measurement $(\mathrm{Pe})$ to evaluate the performance of the proposed algorithm.

$\% \overline{\operatorname{Pr}}$ can be calculated as follows:

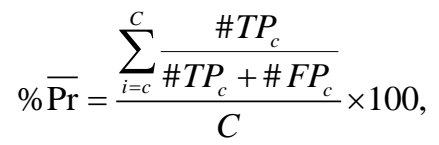

where $C$ is the number of physical activity, $\# T P_{c}$ is the number of the acceleration signals of the $c^{\text {th }}$ activity which are recognized correctly, and $\# F P_{c}$ is the number of the acceleration signals which are recognized to be the $c^{\text {th }}$ activity incorrectly.

The total number of plus and multiply operators used in the wavelet transform, the dimension reduction, and the MLPNN is used to measure the time complexity. Let $l_{s}$, $l_{f}, h$, and $t$ denote the number of the input signal (1248), the filter length, the number of hidden nodes, and output nodes respectively. The number of the operators used in the wavelet transform equals to $4 \times l_{s} \times l_{f}$. The number of the operators used in the proposed dimension reduction equals to $3 \times l_{s}$. The number of the operators used in the MLPNN equals to $2\left(l_{s} / 2 r \times h+h \times t\right)$. Let \#Op $\mathrm{P}_{\max }$ denote the number of operators used in the combination that have the maximum operators. \%Op can be calculated as follows:

$$
\% \mathrm{Op}=\frac{4 \times l_{s} \times l_{f}+3 \times l_{s}+2\left(l_{s} / 2 r \times h+h \times t\right)}{\# \mathrm{Op}_{\max }} \times 100
$$

$\mathrm{Pe}$ is the distance from the point of $\% \overline{\mathrm{Pr}}=100$ and $\% \mathrm{Op}=0$ to the point of $\% \overline{\mathrm{Pr}}$ and $\% \mathrm{Op}$ of the evaluated combination. The lower Pe means the higher performance. Pe can be calculated as follows:

$$
\mathrm{Pe}=\sqrt{(100-\% \overline{\mathrm{Pr}})^{2}+(\% \mathrm{Op})^{2}}
$$

\section{The Experiments}

5 volunteers who were healthy with the ages between 21 to 25 year old were asked to attach the acquisition device at the right side of their waists as shown in Fig. 1. Next, all of the volunteers were asked to perform 5 daily-life activities: 1) walking 2) standing up from a chair 3) sitting down on a chair 4) lying down on a bed and 5) getting up from a bed; and 5 falling events: 1) falling forward 2) falling backward 3) falling to the right side 4) falling to the left side and 5) falling while standing up. Each volunteer was asked to perform each activity 30 times. Then the total number of the acceleration signals of each activity is 150 times. Next, 15 acceleration signals of each activity from each volunteer were randomly selected to calculate $\mathbf{W}$ of the proposed dimension reduction and to train the MLPNN, and the remaining signals were used to test the performance.

The results of the physical activity experiment are shown in Table 3 . Since all of the combinations of the 4 factors are very large, Table 3 . only shows the filter lengths, number of the hidden nodes, $\% \overline{\mathrm{Pr}}, \% \mathrm{Op}$, and $\mathrm{Pe}$ of the combinations which have smallest $\mathrm{Pe}$ for the given mother wavelets and dimension reduction ratios. Moreover in order to compare the results of the proposed dimension reduction, the results of the physical activity recognition which does not use the dimension reduction (n/a) are also shown in Table 3. Also, the combination which has the largest $\% \overline{\mathrm{Pr}}$ is shown at the last row of Table 3.

As shown in Table 3., the proposed dimension reduction not only reduced the $\% \mathrm{Op}$ but also increased the $\% \overline{\mathrm{Pr}}$. Moreover, the optimal combination which has the smallest $\mathrm{Pe}$ was the Biorthogonal wavelet whose filter length is 2 , the proposed dimension reduction whose ratio is 16 , and the MLPNN whose number of hidden nodes is 40 . The $\% \overline{\mathrm{Pr}}$ and $\% \mathrm{Op}$ of the optimal combination were $91.791 \%$ and $5.446 \%$ respectively. The $\% \overline{\mathrm{Pr}}$ and $\% \mathrm{Op}$ of the largest $\% \overline{\mathrm{Pr}}$ combination were $95.124 \%$ and $18.971 \%$ respectively. As a result, the optimal combination has the better trade-off between the accuracy and time complexity. 
Table 3. The summary of the physical activity recognition.

\begin{tabular}{|c|c|c|c|c|c|c|}
\hline Mother Wavelet & Dimension Reduction Ratio & Filter Length & \#Node of Hidden Layer & $\% \overline{\mathrm{Pr}}$ & $\% \mathrm{Op}$ & $\mathrm{Pe}$ \\
\hline \multirow{4}{*}{ Daubechies } & $\mathrm{n} / \mathrm{a}$ & 4 & 26 & 78.517 & 18.971 & 28.661 \\
\hline & 4 & 8 & 28 & 91.711 & 14.543 & 16.740 \\
\hline & 8 & 4 & 38 & 91.125 & 8.903 & 12.571 \\
\hline & 16 & 8 & 38 & 87.777 & 12.023 & 17.145 \\
\hline \multirow{4}{*}{ Coiflet } & $\mathrm{n} / \mathrm{a}$ & 12 & 22 & 79.339 & 25.608 & 32.903 \\
\hline & 4 & 6 & 34 & 90.053 & 13.183 & 16.515 \\
\hline & 8 & 6 & 30 & 92.479 & 10.531 & 12.941 \\
\hline & 16 & 6 & 36 & 78.161 & 9.726 & 23.907 \\
\hline \multirow{4}{*}{ Symlet } & $\mathrm{n} / \mathrm{a}$ & 4 & 30 & 81.718 & 21.207 & 28.000 \\
\hline & 4 & 4 & 30 & 91.149 & 10.393 & 13.651 \\
\hline & 8 & 4 & 34 & 92.631 & 8.608 & 11.331 \\
\hline & 16 & 4 & 40 & 92.309 & 7.664 & 10.858 \\
\hline \multirow{4}{*}{ Biorthogonal } & $\mathrm{n} / \mathrm{a}$ & 6 & 32 & 88.835 & 24.543 & 26.963 \\
\hline & 4 & 2 & 30 & 93.369 & 8.175 & 10.526 \\
\hline & 8 & 2 & 36 & 91.492 & 6.538 & 10.73 \\
\hline & 16 & 2 & 40 & 91.791 & 5.446 & 9.851 \\
\hline Biorthogonal & 16 & 18 & 30 & 95.124 & 22.801 & 23.317 \\
\hline
\end{tabular}

Note that: $\mathrm{n} / \mathrm{a}=$ do not use the proposed dimension reduction.

\section{Conclusions and Future Works}

An efficient physical activity recognition was studied in this paper. The contribution of this paper is twofold. Firstly, this paper proposed a novel dimension reduction technique. The proposed technique not only increase the accuracy of the recognition but also reduce the time complexity. Secondly, this paper carefully studied the combination between the mother wavelets, the filter lengths of the mother wavelet, the dimension reduction ratios, and the number of the nodes in the hidden layer for searching the optimal combination.

In the future works, the additional physical activities such as walking upstairs/downstairs, turning left/right, and 180 degree turning would be experimented. Moreover, the recognition system with the proposed dimension reduction technique would be applied to recognize the physical activities and the falling events of elderly people.

\section{References}

(1) United Nations, Department of Economic and Social Affairs, Population Division : "World Population Ageing 2013", United Nations publication, pp. 8-11, 2013

(2) Centers for Disease Control and Prevention, National Center for Injury Prevention and Control, 2013. Webbased Injury Statistics Query and Reporting System (WISQARS). Available at: http://webappa.cdc.gov/ [Accessed March 4, 2013]

(3) Davide Curone, Gian M. Bertolotti, Andrea Cristiani, Emanuele L. Secco, and Giovanni Magenes : "A RealTime and Self-Calibrating Algorithm Based on Triaxial Accelerometer Signals for the Detechtion of Human Posture and Activity", IEEE Transactions on Information Technology in Biomedicine, Vol. 14, No. 4, pp. 1098-1105, 2010

(4) Yanjun Li, Gan Chen, Yueyun Shen, Yihua Zhu, and Zhen Cheng : "Accelerometer-Based Fall Detection Sensor System for The Elderly", Cloud Computing and Intelligent Systems (CCIS), 2012 IEEE 2nd International Conference on, Vol. 3, pp. 1216-1220, 2012

(5) Sengto A. and Leauhatong T. : "Human falling detection algorithm using back propagation neural network", Biomedical Engineering International Conference (BMEiCON2012), pp. 1-5, 2012

(6) Chun-Lin and Liu : "A Tutorial of the Wavelet Transform", February 2010

(7) A. Phinyomark1, C. Limsakul, and P. Phukpattaranont : "Application of Wavelet Analysis in EMG Feature Extraction for Pattern Classification", Measurement Science Review, Vol. 11, No. 2, pp. 45-52, 2011

(8) Salem and M.A.-M. : "On the selection of the proper wavelet for moving object detection", Computer Engineering \& Systems (ICCES), 2011 International Conference on, pp. 207-212, 2011

(9) S. Patilkulkarni, PhD. Ll, and H C Vijay Lakshmi. : "Vanishing Moments of a Wavelet System and Feature Set in Face Detection Problem for Color Images", International Journal of Computer Applications, Vol. 66, No. 16, pp. 0975 - 8887, 2013

(10) A. J. Jain, R. P. W. Duin, and J. Mao : "Statistical pattern recognition: A review," IEEE Trans. Pattern Analysis and Machine Intelligence, Vol. 22, No. 1, pp. 4-37, 2000

(11) Wuyi Yang : "Regularized Complete Linear Discriminant Analysis for Small Sample Size Problems", ICIC 2012, CCIS 304, pp. 67-73, 2012

(12) YP Chen, JY Yang, SN Liou, GY Lee, and JS Wang : "Online classifier construction algorithm for human activity detection using a tri-axial accelerometer", Applied Mathematics Computation 205(2), pp. 849860,2008

(13) Ercan Ozgan : "Artificial neural network based modelling of the Marshall Stability of asphalt concrete", Expert Systems with Applications 38 (2011), pp. 6025-6030, 2011

(14) Bendi Venkata Ramana, Prof. M.Surendra Prasad Babu, and Prof. N. B. Venkateswarlu : "A Critical Study of Selected Classification Algorithms for Liver Disease Diagnosis", International Journal of Database Management Systems ( IJDMS ), Vol.3, No.2, 2011 